\title{
Efecto de la edad al primer parto sobre parámetros reproductivos en la primera lactancia de vacas Holstein y Jersey de Costa Rica
}

\section{Effect of age at first calving on reproductive parameters in the first lactation of Holstein and Jersey cattle from Costa Rica}

\author{
Gloriana Castillo Badilla $₫$, Mauren Salazar Carranza², Jaime Murillo Herrera ${ }^{3}$, Frank \\ Hueckmann Voss ${ }^{4}$, Juan José Romero Zúñiga ${ }^{5}$
}

1 Cátedra de Salud de Hato y Control de la Producción, Escuela de Medicina Veterinaria, Universidad Nacional, Costa Rica. Apdo.postal: 304-3000 Heredia.E-mail: gloriana.castillo.badilla@una.cr

2 Consultor independiente. Apdo.postal: 304-3000 Heredia. E-mail: mausalazarcarranza@gmail.com

3 Consultor independiente. Apdo. postal: 304-3000 Heredia. E-mail: jmurillo80@gmail.com

4 Consultor independiente. Apdo.postal: 304-3000 Heredia. E-mail: huecsedo@hotmail.com

5 Programa de Investigación en Medicina Poblacional. Escuela de Medicina Veterinaria. Universidad Nacional. Costa Rica. Apdo. Postal: 304-3000 Heredia.E-mail: juan.romero.zuniga@una.cr

Recibido: 04 de Febrero de 2015.Corregido: 29 de Setiembre de 2015. Aceptado: 01 de Noviembre de 2015.

Resumen: Se realizó un estudio retrospectivo en 28367 animales raza Jersey puro y 46029 Holstein puras, de lecherías especializadas de distintas zonas ecológicas de Costa Rica, en el periodo comprendido entre los años 2000 y 2010, con el fin de cuantificar el efecto provocado por la edad al primer parto (EPP) sobre los parámetros reproductivos durante la primera lactancia. Se incluyeron los datos de vacas, registrados en el programa VAMPP Bovino 3.0, en el Centro Regional de Informática para la Producción Animal Sostenible (CRIPAS), de la Escuela de Medicina Veterinaria de la Universidad Nacional de Costa Rica. Los datos fueron analizados por medio de estadística descriptiva y un modelo lineal mixto. Hubo efecto significativo de la EPP sobre el intervalo parto concepción (IPC) en la raza Holstein $(P<0.0001)$, con -13 y -6 días para las hembras con EPP $<26$ meses y EPP 26-33meses, respectivamente. El IPC no se vio afectado, significativamente, por la EPP en la raza Jersey $(P=0.25)$. Asimismo, los servicios por concepción (SPC) correspondieron a 1.44 (D.E. 0.83/IC95\%: $1.44-1.44$ ) para vacas Holstein y 1.48 (D.E. 0.93/IC95\%: $1.46-1.49$ ) para Jersey. Este parámetro se vio influenciado significativamente por la EPP en ambas razas ( $P<0.0001)$; en donde se presentaron 1.1 y 0.6 menos SPC aquellos animales con EPP baja y media, en comparación a los de alta EPP, en la raza Holstein y Jersey respectivamente. De ese modo, el incremento en la EPP, ajustada por variables ambientales, de manejo e intrínsecas de la vaca, tuvo efecto negativo sobre el IPC de la primera lactancia de las vacas Holstein, pero no en Jersey; del mismo modo, hubo un efecto negativo de la EPP sobre los SPC en ambas razas.

Palabras clave: reproducción, bovinos, trópico, salud de hato, sistemas de producción, informática.

Autor de correspondencia: gloriana.castillo.badilla@una.cr

Teléfono: (506) 25624571. 


\begin{abstract}
A retrospective study including 28,367 purebred Jersey and 46,029 purebred Holstein cattle, from specialized dairy farms of different ecological areas in Costa Rica was conducted from 2000 to 2010 in order to quantify the effect of age at first calving (AFC) on the reproductive parameters of the first lactation. Data included records in the VAMPP Bovino 3.0 software from the Regional Information Technology Center for Sustainable Animal Production (CRIPAS) of the School of Veterinary Medicine, National University of Costa Rica. Data was analyzed using descriptive statistics and a linear mixed model. The results showed a significant effect of age at first calving (AFC) in the calving to conception interval (CCI) of Holstein cows ( $P<0.001)$, with -13 and -6 days for cows with AFC $<26$ months and AFC 26-33months, respectively. CCI was not significantly affected in Jersey cows $(P=0.25)$. In addition, average services per conception (SPC) corresponded to 1.44 (D.E. 0.83/IC95\%: 1.44-1.44) for Holstein cows and 1.48 (D.E. 0.93/ IC95\%: $1.46-1.49)$ for Jersey animals. This parameter was significantly influenced by AFC in both breeds $(P<0.0001)$, with a decreased SPC in 1.1 in Holstein and 0.6 in Jersey cows, in those animals with low and medium AFC, compared to high AFC. Consequently, the increment in AFC, adjusted by environmental, management and intrinsic variables of the cow, had a negative effect over the $\mathrm{CCl}$ of the first lactation of Holstein cows, but not in Jersey animals; similarly, there was a negative effect of the AFC over SPC in both breeds.
\end{abstract}

Keywords: reproduction, cattle, tropics, herd health, production systems, Information Technology.

\title{
INTRODUCCIÓN
}

La edad al primer parto (EPP) consiste en el tiempo que tarda un animal en alcanzar su madurez sexual y reproducirse por primera vez (Hare et al. 2006). La EPP puede ser influenciada por el tamaño corporal y el inicio de la actividad hormonal del sistema reproductivo (Moore et al. 1991). Aunque se considera que la edad a la pubertad no está determinada por un peso en particular, sí lo está por un orden indeterminado de condiciones fisiológicas que resultan de un peso dado (Grajales et al. 2006).

La vida productiva de un animal es indicativo de la eficiencia del sistema pecuario, y está influenciado por la EPP, el intervalo entre partos, la duración de cada lactancia y el éxito en soportar otras lactaciones (Hare et al. 2006). La EPP es un importante indicador del desempeño reproductivo del hato, ya que una avanzada edad para el inicio de la vida productiva, constituye una limitante de importancia económica (Bolívar et al. 2009).

Por otro lado, en bovinos lecheros, la búsqueda de mayor eficiencia, tanto biológica como económica, requiere de una elevada producción de leche por lactancia, así como de un buen desempeño reproductivo (Marini et al. 2007). Al respecto, Studer (1998) indica que existe preocupación entre los médicos veterinarios respecto a la habilidad reproductiva de las novillas altas productoras que paren a temprana edad porque podrían experimentar un anestro prolongado.

En estudios realizados con animales Jersey y Holstein de lecherías especializadas de Costa Rica, se determinó la influencia significativa de la EPP sobre los índices productivos y su longevidad dentro del hato (Castillo-Badilla et al. 2013; Salazar-Carranza et al. 2013). Por 
otro lado, el efecto de la EPP sobre los parámetros reproductivos, tales como SPC e IPC, no han sido ampliamente discutidos en la literatura. No obstante, sí se ha relacionado la baja EPP con la presentación de partos distócicos, muy probablemente debido al escaso desarrollo corporal de las terneras (Ettema \& Santos 2004). Por otro lado, se ha concluido que la EPP parece no tener efecto sobre los indicadores reproductivos (Marini et al. 2007).

Una de las posibles relaciones de la EPP con las variables reproductivas es que la reducción de la EPP podría incrementar el número de terneros por vaca, pero podría traer consigo la dificultad de parto con la consecuente reducción en la viabilidad de los terneros, incrementando su susceptibilidad a infecciones debido a una reducida absorción de inmunoglobulinas (Pirlo et al. 2000). Además, conlleva a una reducción significativa de la fertilidad, incrementando los costos asociados a la reproducción, especialmente al intervalo entre partos y al descarte prematuro de animales valiosos (Moore et al. 1990).

Ante las discrepancias observadas sobre este tópico en la literatura, y la ausencia de estudios poblacionales nacionales, este estudio busca documentar el efecto de la EPP sobre los parámetros reproductivos en la primera lactancia de animales puros de las razas Jersey y Holstein de Costa Rica, que son las dos razas predominantes en ese tipo de sistemas de producción.

\section{MATERIALES Y MÉTODOS}

\section{Población de estudio}

Se trabajó con los datos de un total de 28367 vacas Jersey y 46029 Holstein puras, de hatos lecheros especializados de Costa Rica, durante el período 2000-2010, registrados en la base nacional de datos del Centro Regional de Informática para Producción Animal Sostenible, del Programa de Investigación en Medicina Poblacional de la Escuela de Medicina Veterinaria de la Universidad Nacional, que utiliza el paquete informático VAMPP Bovino 3.0 (Noordhuizen \& Buurman 1984; Romero et al. 2000) para la captura y administración de la base de datos en cada finca.

\section{Análisis estadístico}

El análisis de datos se llevó a cabo en varias fases: a) Estadística descriptiva: se realizó el cálculo de medidas de tendencia central (media y mediana) y medidas de dispersión (desviación estándar), ambas para las variables en forma continua; asimismo, se calcularon porcentajes para las variables discretas. Para ambos tipos de medidas se determinó el intervalo de confianza al 95\% (IC 95\%). b) Estimación del efecto de la EPP sobre los parámetros reproductivos de la primera lactancia. Se realizó mediante un modelo múltiple lineal mixto, en el cual se creó un modelo para la evaluación de cada variable dependiente (intervalo parto-concepción y servicios por concepción). 
El modelo estadístico para el efecto sobre el intervalo parto concepción fue:

$$
Y_{i j k l m n o}=\mu O+e p p_{i}+h_{a e_{j}}+\operatorname{lac}_{k}+e \mathrm{eco}_{l}+\text { part }_{m}+\text { endo }_{n}+\text { ret }_{o}+\text { eijklmno }
$$

Donde:

Yijklmno= Intervalo parto concepción

$\mu O=$ Media general

$e p p_{i}=$ Efecto fijo $i$-ésimo de la edad al primer parto (meses)

$h a e_{j}=$ Efecto aleatorio $j$-ésimo del hato/año/época de parto

$l a c_{k}=$ Efecto fijo de $k$-ésimo producción láctea (kg 305d) en la primera lactancia

eco $=$ Efecto fijo de $l$-ésimo de la ecozona $(l=1-9)$ (Holdridge 1987)

part $_{m}=$ Efecto fijo de $m$-ésima del tipo de parto

endo $_{n}=$ Efecto fijo de $n$-ésima del coeficiente de endogamia

ret $t_{0}=$ Efecto fijo de $o$-ésima de la retención de membranas fetales

eijklmno= Efecto aleatorio residual

El modelo estadístico para el efecto sobre el número de servicios por concepción fue:

$Y_{i j k l m n o}=\mu \mathrm{O}+e p p_{i}+$ hae $_{j}+\mathrm{lac}_{\mathrm{k}}+e \mathrm{co}_{l}+$ part $_{m}+$ endo $_{n}+$ ret $_{o}+$ eijklmno

Donde:

Yijklmno= Servicios por concepción

$\mu O=$ Media general

$e p p_{i}=$ Efecto fijo $i$-ésimo de la edad al primer parto (meses)

$h a e_{j}=$ Efecto aleatorio $j$-ésimo del hato/año/época de parto

$l a c_{k}=$ Efecto fijo de k-ésimo producción láctea (kg 305d) en la primera lactancia

eco= Efecto fijo de $l$-ésimo de la ecozona $(l=1$ - 9) (Holdridge 1987)

part $_{m}=$ Efecto fijo de $m$-ésima del tipo de parto

endo ${ }_{n}=$ Efecto fijo de $n$-ésima del coeficiente de endogamia

ret $t_{0}=$ Efecto fijo de $o$-ésima de la retención de membranas fetales

eijklmno= Efecto aleatorio residual

Los análisis estadísticos se realizaron con el programa SAS versión 9.2 (SAS Institute Inc., SAS@), específicamente PROC UNIVARIATE y PROC MIXED. 


\section{RESULTADOS}

El promedio de la EPP fue 30.7 meses (D.E:6.8/IC 95\%:30.6-30.8) y durante el periodo se presentó una variación entre entre los 30 y 31 meses. Por otra parte, la EPP promedio para las vacas Jersey fue de 29.35 meses (DE: 6.77/IC95\%: 29.27 - 29.42), con una tendencia al incremento con el transcurso de los años. El detalle de la descripción de este parámetro en la raza Holstein se puede observar en Salazar-Carranza et al. (2013), y para las Jersey en Castillo-Badilla et al. (2011).

En forma general se determinó que, para la raza Holstein, el promedio del intervalo parto concepción (IPC) fue de 148.73 (D.E. 95.05/IC95\%: 147.67-149.78) y, de 117.92 (D.E. 81.36/ IC95\%: 116.86 - 118.87), para la raza Jersey. Por otro lado, los servicios por concepción (SPC) correspondieron a 1.44 (D.E. 0.83/IC95\%: $1.44-1.44$ ) para vacas Holstein y 1.48 (D.E. 0.93/IC95\%: 1.46 - 1.49) para Jersey.

\section{Efecto de la edad al primer parto sobre el intervalo parto concepción}

La EPP presentó un efecto significativo en la raza Holstein; así, las vacas con EPP < 26 meses presentaron -13.4 días, respecto a las de EPP $>33.6$ meses. De igual forma, en las vacas que paren a una edad intermedia (26.0 -33.6 meses), el IPC fue 8 días menor (Cuadro 1).

Cuadro 1. Efecto de la edad al primer parto, ajustado por variables de tiempo, lugar y animal, sobre el intervalo parto-concepción de la primera lactancia de vacas Holstein de lechería especializada de Costa Rica.

\begin{tabular}{llllll}
\hline Variable & Estrato & Estimado & Diferencia & EE* & P** \\
\hline Intercepto & & & 201.10 & 12.12 & $<0.0001$ \\
\hline EPP (meses) & $<26.0$ & 153.17 & -13.37 & 3.14 & $<0.0001$ \\
\hline & $26.0-33.6$ & 158.43 & -8.12 & 2.86 & 0.0046 \\
\hline Zona & $>33.6$ & 166.55 & 0 & 0 & 0 \\
\hline & bh-MB & 150.38 & -28.89 & 8.52 & 0.007 \\
\hline & bh-P & 145.37 & -33.90 & 8.33 & $<0.0001$ \\
\hline & bmh-P & 157.71 & -21.56 & 8.87 & 0.0151 \\
\hline & bmh-T & 158.27 & -21.00 & 8.20 & 0.0105 \\
\hline bp-MB & 168.10 & -11.17 & 8.27 & 0.1769 \\
\hline bp-P & 156.61 & -22.66 & 8.41 & 0.0071 \\
\hline kg3051***: & bmh-M & 179.27 & 0 & 0 & 0 \\
\hline & $<3948$ & 159.77 & -6.50 & 2.61 & 0.0128 \\
\hline & $3948-6386$ & 152.12 & -14.15 & 2.30 & $<0.0001$ \\
\hline & $>6386$ & 166.27 & 0 & 0 & 0 \\
\hline
\end{tabular}

$* \mathrm{EE}=$ error estándar de la diferencia. $* * \mathrm{P}=$ valor de $\mathrm{P}$ de la diferencia. *k:kg305d: producción corregida a 305 días.

bh-MB: bosque húmedo-Montano Bajo; bh-P: bosque húmedo-Premontano; bmh-P: bosque muy húmedo-Premontano; bmh-T: bosque muy húmedoTropical; bp-MB: bosque pluvial-Montano Bajo; bp-P: bosque pluvialPremontano; bp-MB: bosque pluvial-Montano. 
Este efecto no se observó en la raza Jersey, en la cual no se presentaron diferencias significativas según la EPP (Cuadro 2).

Cuadro 2. Efecto de la edad al primer parto, ajustado por variables de tiempo, lugar y animal, sobre el intervalo parto-concepción de la primera lactancia de vacas Jersey de lechería especializada de Costa Rica.

\begin{tabular}{|c|c|c|c|c|c|}
\hline Variable & Estrato & Estimado & Diferencia & E.E* & $\mathrm{P} * *$ \\
\hline Intercepto & & 132.92 & & 16.13 & $<0.0001$ \\
\hline \multirow[t]{3}{*}{ EPP } & $<25.8$ & 114.48 & -3.68 & 3.25 & 0.2569 \\
\hline & $25.8-31.0$ & 115.34 & -2.82 & 3.11 & 0.3669 \\
\hline & $>31.0$ & 118.17 & 0 & 0 & 0 \\
\hline \multirow[t]{7}{*}{ Zona } & bh-MB & 117.31 & -11.08 & 8.02 & 0.1671 \\
\hline & bh-P & 104.54 & -23.85 & 7.99 & 0.0029 \\
\hline & bmh-P & 100.59 & -27.80 & 8.16 & 0.0007 \\
\hline & bmh-T & 115.59 & -12.81 & 7.89 & 0.1048 \\
\hline & bp-MB & 128.04 & -0.35 & 7.91 & 0.9640 \\
\hline & bp-P & 117.53 & -10.87 & 8.41 & 0.1964 \\
\hline & bmh-M & & 0 & 0 & 0 \\
\hline \multirow[t]{3}{*}{$\operatorname{kg} 305 d^{\prime * 2 * ;}$} & $<2400$ & 119.68 & 1.13 & 4.47 & 0.8005 \\
\hline & $2400-4302$ & 109.76 & -8.78 & 2.04 & $<0.0001$ \\
\hline & $>4302$ & 118.55 & 0 & 0 & 0 \\
\hline
\end{tabular}

*EE= Error estándar de la diferencia. **:P= Valor de $\mathrm{P}$ de la diferencia. **'kg305d: producción corregida a 305 días.

bh-MB: bosque húmedo-Montano Bajo; bh-P: bosque húmedo-Premontano; bmh-P: bosque muy húmedo-Premontano; bmh-T: bosque muy húmedo-Tropical; bp-MB: bosque pluvial-Montano Bajo; bp-P: bosque pluvial-Premontano; bp-MB: bosque pluvial-Montano.

Además del efecto ajustado de la EPP sobre el IPC de la primera lactancia en la raza Holstein, se pudo evidenciar que la zona ecológica y el nivel de producción tuvieron un efecto significativo en ambas razas (Cuadro 1 y 2). Así, en el caso de la Holstein, tomando como base de comparación la zona bmh-M, todas las demás ecozonas presentaron menor IPC (Cuadro 1). Por otro lado, las zonas bh-P y bmh-P mostraron variaciones significativas de -23 y -27 días, que el área bmh-M para la raza Jersey (Cuadro 2).

Adicionalmente, tomando como referencia las vacas con una mayor producción a 305d, en la raza Holstein, tanto las vacas de producción intermedia como las de menor producción presentan una diferencia significativa de $-14.15(p<0.0001)$ y $-6.50(p=0.012)$ días de IPC 
(Cuadro 1). Situación diferente sucedió en las Jersey, en donde las de producción intermedia (2400-4302 kg 305 d) presentaron -8 días $(P<0.0001)$, pero las de baja producción $(<2400$ kg 305 d) presentaron +1.13 días $(P=0.80$ ) (Cuadro 2).

Las hembras con un parto normal tuvieron una disminución no significativa del IPC de 13 días en vacas Holstein y 5.2 días en Jersey, comparando con las que tuvieron un parto distócico (Holstein $p=0.45 /$ Jersey $p=0.34$ ). De igual manera, en ambas razas no hubo efecto significativo de la retención de membranas fetales (RMF) sobre el IPC (Jersey: $P=0.85$ / Holstein: $p=0.60$ ). Similar situación ocurrió con el coeficiente de endogamia (CE), en que no hubo efecto en ninguna de las dos razas (Cuadro 1 y 2 ).

\section{Efecto de la edad al primer parto sobre el número de servicios por concepción}

Los servicios por concepción (SPC) se vieron afectados significativamente por la EPP; así, hembras con una EPP baja (<25.8 meses) y media (25.8-31.0 meses), presentaron menos servicios que aquellas vacas con una alta EPP (>31.0 meses), siendo -1.1 y -0.6 en Holstein y $-1.7 y-1.2$ en Jersey respectivamente $(P<0.0001)$ (Cuadro 3 y 4$)$.

De igual manera, la ecozona afectó significativamente los SPC en ambas razas. Utilizando como base de comparación la zona bmh-M, en las Holstein solo se determinó un efecto significativo en la ecozona correspondiente al bmh-P $(p=0.018)$, mientras que en las demás ecozonas se presentaron variaciones no significativas (Cuadro 3). Asimismo, en vacas Jersey se observó que únicamente las vacas de la ecozona bp-P presentaron +0.33 SPC ( $\mathrm{P}<0.0001)$ (Cuadro 4).

Finalmente, en ambas razas, la producción de leche a 305d en la primera lactancia, afectó significativamente los SPC, tanto en la producción baja, como la media, en que se registraron -0.18 y -0.15 SPC $(P<0.0001)$ para la raza Holstein $y-0.08$ y -0.11 para Jersey, respectivamente $(P=0.01 ; P<0.0001)$, en comparación con las altas productoras (Cuadro 3 y 4 ).

Finalmente, el tipo de parto y el coeficiente de endogamia no tuvieron efecto significativo sobre los SPC de la primera lactancia en ninguna de las dos razas. 
Cuadro 3. Efecto de la edad al primer parto, ajustado por variables de tiempo lugar y animal, sobre el número de servicios por concepción de la primera lactancia de vacas Jersey de lechería especializada de Costa Rica.

\begin{tabular}{llllll}
\hline Variable & Estrato & Estimado & Diferencia & EE* & P** \\
\hline Intercepto & & & 2.31 & 0.10 & $<0.0001$ \\
\hline EPP (meses) & $<26$ & 1.08 & -1.11 & 0.02 & $<0.0001$ \\
\hline & $26-33.6$ & 1.56 & -0.64 & 0.02 & $<0.0001$ \\
\hline Zona & $>33.6$ & 2.20 & 0 & 0 & 0 \\
\hline & bh-MB & 1.75 & 0.13 & 0.07 & 0.0869 \\
\hline & bh- P & 1.61 & -0.01 & 0.07 & 0.8091 \\
\hline & bmh-P & 1.43 & -0.18 & 0.07 & 0.0178 \\
\hline & bmh-T & 1.65 & 0.03 & 0.07 & 0.6754 \\
\hline & bp-MB & 1.55 & -0.07 & 0.07 & 0.3415 \\
\hline bp-P & 1.66 & 0.03 & 0.07 & 0.6245 \\
\hline kg305d & bmh-M & 1.62 & 0 & 0 & 0 \\
\hline & $<3948$ & 1.54 & -0.18 & 0.02 & $<0.0001$ \\
\hline & $3948-6386$ & 1.57 & -0.15 & 0.02 & $<0.0001$ \\
\hline & $>6386$ & 1.73 & 0 & 0 & 0 \\
\hline
\end{tabular}

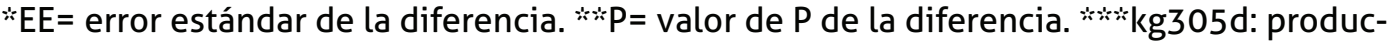
ción corregida a 305 días.

bh-MB: bosque húmedo-Montano Bajo; bh-P: bosque húmedo-Premontano; bmh-P: bosque muy húmedo-Premontano; bmh-T: bosque muy húmedo-Tropical; bp-MB: bosque pluvialMontano Bajo; bp-P: bosque pluvial-Premontano; bp-MB: bosque pluvial-Montano. 
Cuadro 4. Análisis multivariado para el efecto de las variables de tiempo, lugar y animal, sobre los servicios por concepción de la primera lactancia Raza Jersey.

\begin{tabular}{|c|c|c|c|c|c|}
\hline Variable & Estrato & Estimado & Diferencia & E.E* & $P: *$ \\
\hline Intercepto & & 3.24 & & 0.18 & $<0.0001$ \\
\hline \multirow[t]{3}{*}{ Edad al parto } & $<25.8$ & 1.29 & -1.70 & 0.04 & $<0.0001$ \\
\hline & $25.8-31.0$ & 1.87 & -1.19 & 0.03 & $<0.0001$ \\
\hline & $>31.0$ & 3.06 & 0 & 0 & 0 \\
\hline \multirow[t]{7}{*}{ Zona } & bh-MB & 2.22 & 0.23 & 0.10 & 0.0219 \\
\hline & bh-P & 2.05 & 0.07 & 0.10 & 0.4753 \\
\hline & bmh-P & 1.97 & -0.005 & 0.10 & 0.9575 \\
\hline & bmh-T & 2.11 & 0.12 & 0.10 & 0.2151 \\
\hline & bp-MB & 1.88 & -0.10 & 0.10 & 0.3196 \\
\hline & bp-P & 2.31 & 0.33 & 0.10 & 0.0022 \\
\hline & bmh-M & 1.98 & 0 & 0 & 0 \\
\hline \multirow[t]{3}{*}{ Kg305d } & $<2400$ & 2.06 & -0.08 & 0.03 & 0.0179 \\
\hline & $2400-4302$ & 2.02 & -0.11 & 0.02 & $<0.0001$ \\
\hline & $>4302$ & 2.14 & 0 & 0 & 0 \\
\hline
\end{tabular}

*EE= Error estándar de la diferencia; **P= Valor de $\mathrm{P}$ de la diferencia; ***Kg305d: producción corregida a 305 días.

bh-MB: bosque húmedo-Montano Bajo; bh-P: bosque húmedo-Premontano; bmh-P: bosque muy húmedo-Premontano; bmh-T: bosque muy húmedo-Tropical; bp-MB: bosque pluvial-Montano Bajo; bp-P: bosque pluvial-Premontano; bp-MB: bosque pluvial-Montano.

\section{DISCUSIÓN}

En este estudio fue posible documentar el efecto de la EPP sobre el IPC y los SPC de la primera lactancia, ajustado por variables de ambiente y manejo. En el caso de la Holstein, se logró determinar un efecto importante en aquellos animales con EPP bajos $>25$ meses, contrario a lo que reportan (Hussein \& El Agawany 2009), quienes asocian la disminución de la EPP con valores mayores en la IPC.

En contraste, la EPP no presentó efecto significativos sobre el IPC en las Jersey, concordando con lo reportado para animales en condiciones de pastoreo, en donde no se observó efecto marcado de la EPP sobre los indicadores reproductivos (Bouissou 1997; Marini et al. 2007). 
La zona ecológica, es una variable de peso, ya que esta influye tanto en las variables productivas como en las reproductivas (Castillo-Badilla et al. 2013; Salazar-Carranza et al. 2013), reafirmando la implicación del ambiente, temperatura, humedad y radiación solar en el rendimiento productivo y reproductivo de los animales en lecherías tropicales.

Por otra parte, los animales cuya producción a $305 \mathrm{~d}$ fue baja y media, mostraron un IPC menor que las vacas con una mayor producción, concordando con lo observado en estudios anteriores, los cuales reportan asociaciones positivas entre la producción de leche y los indicadores reproductivos. Se indica que las novillas con mayor producción muestran un deterioro en su capacidad de reiniciar la actividad reproductiva luego del primer parto (Kim \& Suh 2003; Marini et al. 2007). Igualmente. Hussein \& El Agawany (2009) asociaron alteraciones en el postparto con una alta producción de leche, especialmente en vacas que paren a edad media ( $\geq 24$-28 meses) y alta (> 28 meses), lo cual podría indicar la alta predisposición de las vacas con una producción superior a presentar desordenes postparto (Roche et al. 2013).

Los SPC, se ven disminuidos, conforme se disminuye la EPP $(P<0.0001)$; sin embargo, las vacas con bajas EPP son las de menores niveles de producción, por lo tanto un menor compromiso metabólico. Estos resultados se confirman con previos reportes, en donde aquellas hembras con EPP <23meses obtuvieron mejores índices reproductivos, presentando una disminución de los SPC, y mayor longevidad dentro del hato (Cooke et al. 2013; Zavadilová \& Stipková 2013). Este dato se puede relacionar a la demanda generada por la gestación y la lactancia, en donde aquellos animales altos productores tienen mayor predisposición a sufrir enfermedades metabólicas y por lo tanto retrasar su retorno a ciclicidad (Wathes et al. 2007; Roche et al. 2013)

Asimismo, los animales que presentaron RMF, tuvieron mayor cantidad de SPC (2.26 servicios), que aquellas con parto normal, información que concuerda con lo reportado por Lozano-Domínguez (1999), que determinó que hembras que presentaron RMF o metritis postparto, tuvieron un servicio más que aquellas que no exhibieron ningún tipo de patología en el postparto.

Tal como se describe ampliamente en la literatura, los animales con mayor nivel productivo presentaron un incremento en el número de servicios. Esto representa uno de los mayores problemas y retos que enfrenta la industria láctea en los últimos años, en que los animales altos productores tienen un pobre desempeño reproductivo; todo esto provocado por distintos factores fisiológicos, ambientales, nutricionales y de manejo (Lucy 2001; Pryce et al. 2004; Roche et al. 2013).

Finalmente, en total concordancia con los hallazgos de investigaciones recientes (McParland et al. 2007; Rokouei et al. 2010), el tipo de parto y el coeficiente de endogamia no influyeron en los SPC de población estudiada. 


\section{CONCLUSIONES}

El incremento en la EPP, ajustada por variables ambientales, de manejo e intrínsecas de la vaca, tuvo efecto negativo sobre el IPC de la primera lactancia de las vacas Holstein, lo que no ocurrió en las Jersey. Asimismo, se estableció un efecto negativo -significativode la EPP sobre los SPC en ambas razas. Si se conoce que un incremento en la EPP puede significar una vida productiva menos eficiente dentro del hato, un aumento en el IPC y los SPC suman efectos negativos para la salud del hato.

\section{REFERENCIAS}

Bolívar, D., Echeverry, J., Restrepo, L. \& Cerón-Muñóz, M. 2009. Productividad de vacas Jersey, Holstein y Jersey/Holstein en una zona de bosque húmedo montano bajo (Bh-MB) [en línea]. Livestock Research for Rural Development. http://www.lrrd.org/ Irrd21/6/boli21080.htm. (Consulta: 24 ene. 2011)

Bouissou, R.G. 1997. Edad al primer parto. Marca Líquida. 64: 41-44.

Castillo-Badilla, G., Salazar-Carranza, M., Murillo-Herrera, J. \& Romero-Zúñiga, J.J. 2011. Factores que afectan la edad al primer parto en vacas Jersey de -lechería especializada en Costa Rica. Rev. Ciencias Veterinarias. 29: 7-19.

Castillo-Badilla, G., Salazar-Carranza, M., Murillo-Herrera, J., Hueckmann-Voss, F. \& RomeroZúñiga, J.J. 2013. Efecto de la edad al primer parto sobre la producción láctea en vacas Jersey de lechería especializada de Costa Rica. Agronomía Mesoamericana (Costa Rica). 24: 177-187. doi: 10.15517/am.v24i1.9796

Cooke, J.S., Cheng, Z., Bourne, N.E. \& Wathes, D. C. 2013. Association between growth rates, age at first calving and subsequent fertility, milk production and survival in HolsteinFriesian heifers. Open Journal of Animal Sciences 3: 1-12. [en línea]. http://dx.doi. org/10.4236/ojas.2013.31001 (Consulta: 6 ene. 2014). doi: 10.4236/ojas.2013.31001

Ettema, J. \& Santos, J. 2004. Impact of age at first calving on lactation, reproduction, health, and income in First-Parity Holstein on Commercial Farms. J.Dairy Sci. 87: 2730-2742. doi:10.3168/jds.S0022-0302(04)73400-1

Grajales, H., Hernández, A. \& Prieto, E. 2006. Edad y peso a la pubertad y su relación con la eficiencia reproductiva de grupos raciales bovinos en el trópico colombiano. [en línea].http://www.lrrd.org/lrrd18/10/graj18139.htm. (Consulta: 3 nov.2011).

Hare, E., Norman, H. \& Wright, J. 2006. Trends in calving age and calving intervals for Dairy cattle breeds in the United States. J. Dairy. Sci. 89: 365-370. doi: 10.3168/jds.S00220302(06)72102-6

Holdridge. L. R. 1987. Ecología basada en zonas de vida. IICA. San José. C. R. 219p. 
Kim. I.H. \& G.H. Suh. 2003. Effect of the amount of body condition loss from the dry to near calving periods on the subsequent body condition change, occurrence of postpartum diseases, metabolic parameters and reproductive performance in Holstein dairy cows. Theriogenology. 60. 1445-1456. doi: 10.1016/S0093-691X(03)00135-3

Lozano-Dominguez, R.R. 1999. Problemas en el periparto y su consecuencias reprodcutivas en vacas lecheras. [en línea].Inifap.HYPERLINK "http://www.inifapaguascalientes. gob.mx/Publicaciones"http://www.inifapaguascalientes.gob.mx/Publicaciones. (Consulta: 25 oct. 2013)

Lucy, M.C. 2001. Reproductive Loss in High-Producing Dairy Cattle: Where Will It End? J. Dairy Sci. 84:1277-1293. doi: 10.3168/jds.S0022-0302(01)70158-0

Marini, P.R., Charmandarian, A. \& Di Masso, R.J. 2007. Desempeño productivo y reproductivo de vacas de diferentes edades al primer parto en sistemas a pastoreo [en línea]. Sitio Argentino de Producción Animal. http://www.produccion-animal.com.ar/. (Consulta: 25 feb.2011)

McParland, S.M., Kearney, J.F., Rath, M. \& Berry, D.P. 2007. Inbreeding effects on milk production calving performance fertility and conformation in Irish Holstein-Friesians. J. Dairy Sci., 90:4411-4419. doi: 10.3168/jds.2007-0227

Moore, R.K., Kennedy, B.W., Schaeffer, L.R. \& Moxley, J.E. 1991. Relationships between age and body weight at calving and production in first lactation Ayrshires and Holsteins. J. Dairy Sci. 74:269-278. doi: 10.3168/jds.S0022-0302(91)78170-8

Noordhuizen, J.P.T.M. \& Buurman, J. 1984. Veterinary automated management and production control program for dairy farms (VAMPP), the application of MUMPS for data processing. Vet. Quarterly. 6:62-77.

Pirlo, G., Miglior, F. \& Speroni, M. 2000. Effect of age at first calving on production traits and on difference between milk yield returns and rearing costs on Italian Holstein. J. Dairy Sci., 83: 603-608. doi: 10.3168/jds.S0022-0302(00)74919-8

Pryce, J.E., Royal, M.D., Garnsworthy, P.C. \& Mao, I.L. 2004. Fertility in the high-producing dairy cow. Livestock Production Sci., 86: 125-135. doi: 10.1016/S0301-6226(03)00145-3

Roche, J.R., Kay, J.K., Friggens, N.C., Loor, J. J. \& Berry, D.P. 2013. Assessing and Managing Body Condition Score for the Prevention of Metabolic Disease in Dairy Cows. Vet Clin Food Anim 29: 323-336. doi: 10.1016/j.cvfa.2013.03.003

Rokouei, M., Vaez-Torshimi, R., Moradi-Shahrbabak, M., Sargolzaei, M. \& Sorensen, A.C. 2010. Monitoring inbreeding trends and inbreeding depression for economically important traits of Holstein cattle in Iran. J. Dairy Sci., 93:3294-3302. doi: 10.3168/jds.2009-2748

Romero, JJ., Estrada, S. \& Pérez, E. 2000. Uso del sistema de información VAMPP en investigaciones a gran escala. XVII Congreso Panamericano de Ciencias Veterinarias. 11-15 Setiembre.101p. 
Salazar-Carranza, M., Castillo-Badilla, G., Murillo-Herrera, J., Hueckmann-Voss, F. \& RomeroZúñiga, J.J. 2013. Edad al primer parto en vacas Holstein de lechería especializada en Costa Rica. Revista Agronomía Mesoamericana (Costa Rica). 24(2): 1-11. doi: 10.15517/ am.v24i2.12522

SAS Institute Inc., SAS®. User's Guide. Version 6. 1990. 4th edition. Cary. NC. USA. SAS Institute Inc. 943 pp.

Studer, E. 1998. A veterinary perspective of on-farm evaluation of nutrition and reproduction. J. Dairy Sci., 81:872-876. doi: 10.3168/jds.S0022-0302(98)75645-0

Wathes, D.C., Fenwick, M., Cheng, Z., Bourne, N., Llewellyn, S., Morris, D.G., Kenny, D., Murphy, J. \& Fitzpatrick, R. 2007. Influence of negative energy balance on cyclicity and fertility in the high producing dairy cow. Theriogenology. 68S: S232-S241. doi: 10.1016/j. theriogenology.2007.04.006

Zavadilová, L. \& Štípková, M. 2013. Effect of age at first calving on longevity and fertility traits for Holstein cattle. Czech J. Anim. Sci., 58: 47-57. 